\title{
INTERPRETING STUDENT RESPONSES USING SENTIMENT ANALYSIS AND TEXT-ANALYTICS
}

\author{
Madison Mindorff, Brendan Lobo, Chirag Variawa \\ University of Toronto \\ madison.mindorff@mail.utoronto.ca, b.lobo@mail.utoronto.ca, chirag.variawa@utoronto.ca
}

\begin{abstract}
This paper discusses exploratory research which computationally examines over one and a half million words presented by first-year students as part of weekly online assignments over the Fall 2020 academic term. This work aims to explore whether computational analyses of first-year engineering student vocabulary can be employed to understand the levels of student motivation when learning engineering in an online environment. The investigation uses NVivo 12 Plus (NVivo), a data analysis software, to track the overall sentiment of weekly student discussion board responses and apply text queries to determine the number of responses that include words related to the expectancy-value theory. Applying this theory reveals trends in overall student motivation, with weeks four to six and eight to ten having an overall positive sentiment. This positive sentiment reveals higher levels of student motivation during those weeks.
\end{abstract}

Keywords: sentiment analysis, motivation, online learning, text analytics, engineering education, engineering, firstyear engineering,

\section{INTRODUCTION}

\subsection{Background}

Due to the impact COVID-19 has on the contemporary educational landscape, and courses being moved online, methods of teaching and assessment used in an online context have become more prevalent. One of these methods includes the use of online discussion boards. An example of this is APS100 ( $\mathrm{n}=1100$ students), a mandatory course for all first-year undergraduate engineering students at the University of Toronto. This course supports students' transition into first-year engineering studies by allowing them to develop and apply a greater understanding of the academic learning environment, the professional skills needed for success, and the professional nature of engineering [1]. The mandatory weekly discussion prompts aim to encourage reflection on academic/professional development. Each week students were given four prompts to select from, and responses were collected over the Fall 2020 academic term.
These responses were then computationally examined using Natural Language Processing (NLP).

\subsection{Motivation}

Existing literature suggests that online learning can be especially stressful for students, having an impact on their sentiment and appreciation of a course [2]. Currently, there is a scarce amount of computational surveying that is being done longitudinally (i.e. from the beginning to the end of a course) that shows how student sentiment changes in a first-year Canadian engineering education context. The gap in this research is the motivation for this work. By analyzing the sentiment of student responses to weekly online discussion boards, patterns in student motivation can be identified.

NLP is a type of artificial intelligence that analyzes the human language and allows computers to read and interpret text, measure sentiment, and categorize unstructured data [3] [4]. To identify patterns, trends, and/or prevailing themes from large volumes of data, natural language processing and text analytics are used together. As well, sentiment analysis, a sub-field of NLP, is used to identify the mood of the subject's work and define it as either positive, negative, or neutral [3]. For this research, NVivo 12 Plus (NVivo), a software which uses NLP is used to analyze response sentiment and words related to motivation, drawn from the Expectancy-Value Theory (EVT). This theory proposes that if a student expects to be successful in completing a task and believes it is worth doing, they will have more motivation. The reason for using natural language processing is the challenge of removing human bias and error when organizing and analyzing the data manually. As well, with over one and a half million words in the data set, the time and cost to conduct this work manually is infeasible. Using natural language processing allows for large volumes of unstructured data to be interpreted, categorized, and classified by sentiment, consistently without bias, in minimal time [3]. 


\subsection{Problem Definition}

The focus of this research surrounds the question: Can computational analysis of first-year engineering student vocabulary be employed to understand the levels of student motivation when learning engineering in an online environment?

\subsection{Solutions Considered}

Any approach that attempts to answer this question needs to abide by certain conditions. One condition imposed by the very nature of the question is that the solution needs to be able to digest student-generated communications. The communication can be textual or verbal, but it must be thematically diverse in order to avoid inherent bias in the results. For example, the data used cannot solely be focused on what makes students demotivated or vice-versa. Another bias combatting condition is that the data set needs to be generated by a student body with the size and diversity to adequately represent the first-year engineering population. This helps to account for individuals with exceptionally high or low levels of motivation. Finally, the theory by which the motivation levels are evaluated needs to be compatible with the limits of the analysis software and computational hardware available.

Textual student responses were chosen as the data type to be examined as they are more readily available than verbal communications and are easier to process in large quantities compared to hard written responses. The data was collected from the core course, APS100, rather than other sources for two reasons. The first being that it is a mandatory course for all first-year engineering students. Since it encompasses the entire first-year engineering student population, its size and diversity are reflective of said population. The second reason for the selection of this course is that it mandated thematic diversity. Students were required to respond to one of four discussion topics each week. These topics covered different aspects of that week's curriculum (i.e. concepts taught in the course) and student life (e.g., midterms, reading week, personal experiences, etc.). These questions would correspond to a general theme and this theme would change on a weekly basis. To analyze this dataset, motivational theories featured in M. Svinicki's publication "A Guidebook On Conceptual Frameworks For Research In Engineering Education" [5] are taken into consideration. The conclusion from this discussion is that the Expectancy-Value Theory [6] and the SelfDetermination Theory [7] are both viable candidates in this analysis due to their broad understanding of motivation [5] and their conduciveness to being implemented with computational analysis. This investigation uses the Expectancy-Value Theory due to its relative simplicity in terms of implementation compared to the SelfDetermination Theory.

\subsection{Theoretical Framework}

Motivation is the driving force behind human actions [8]. It is the willingness to complete a task or achieve a goal [9]. There are many motivational theories which aim to explain the drive behind individuals' actions to complete or achieve a particular goal [10]. The framework used for this research is the Expectancy-Value Theory developed by Eccles-Parsons and her colleagues (1983). They proposed that expectancies and values directly impact an individual's performance [11]. In the engineering education context, this theory proposes that if a student expects to be successful in completing a task and believes it is worth doing, they will have more motivation [5].

\subsection{Selected Methodologies}

NVivo was selected due to the cost limitations and the technical capabilities of the researchers. NVivo requires minimal programming knowledge while providing the necessary features for this work, including sentiment analysis and text search queries.

\section{METHODOLOGY}

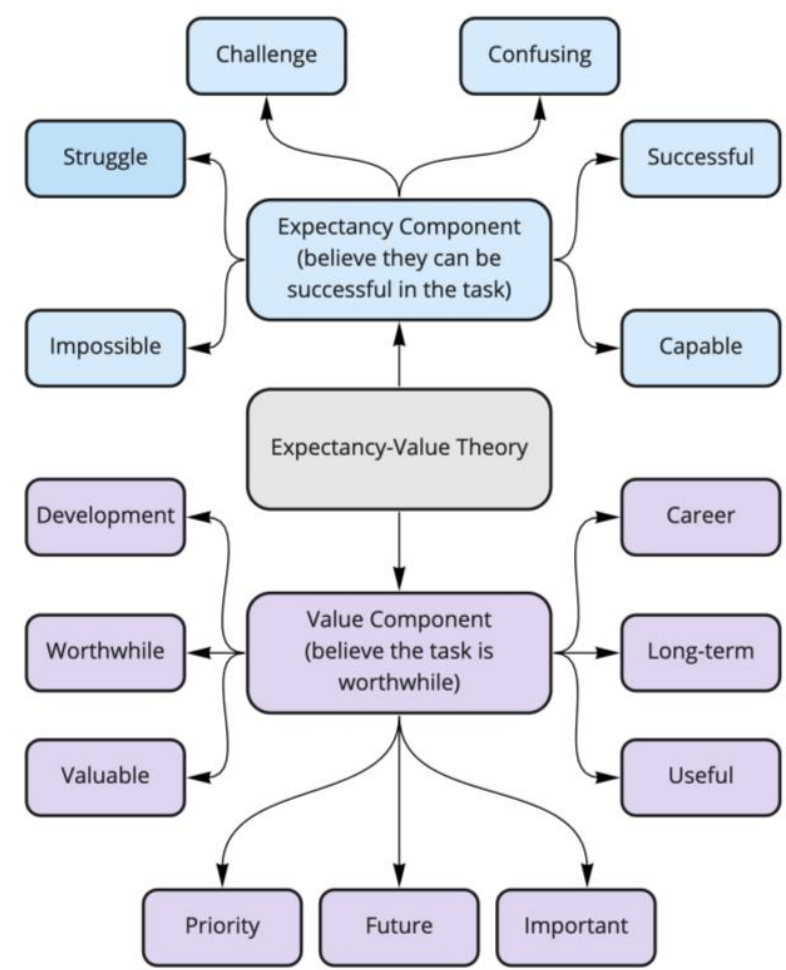

Fig. 1. This figure shows which words were used when analyzing the value and expectancy components of motivation. These words and their synonyms can be found in Svinicki's “A Guidebook On Conceptual Frameworks For Research In Engineering Education” [5]. 


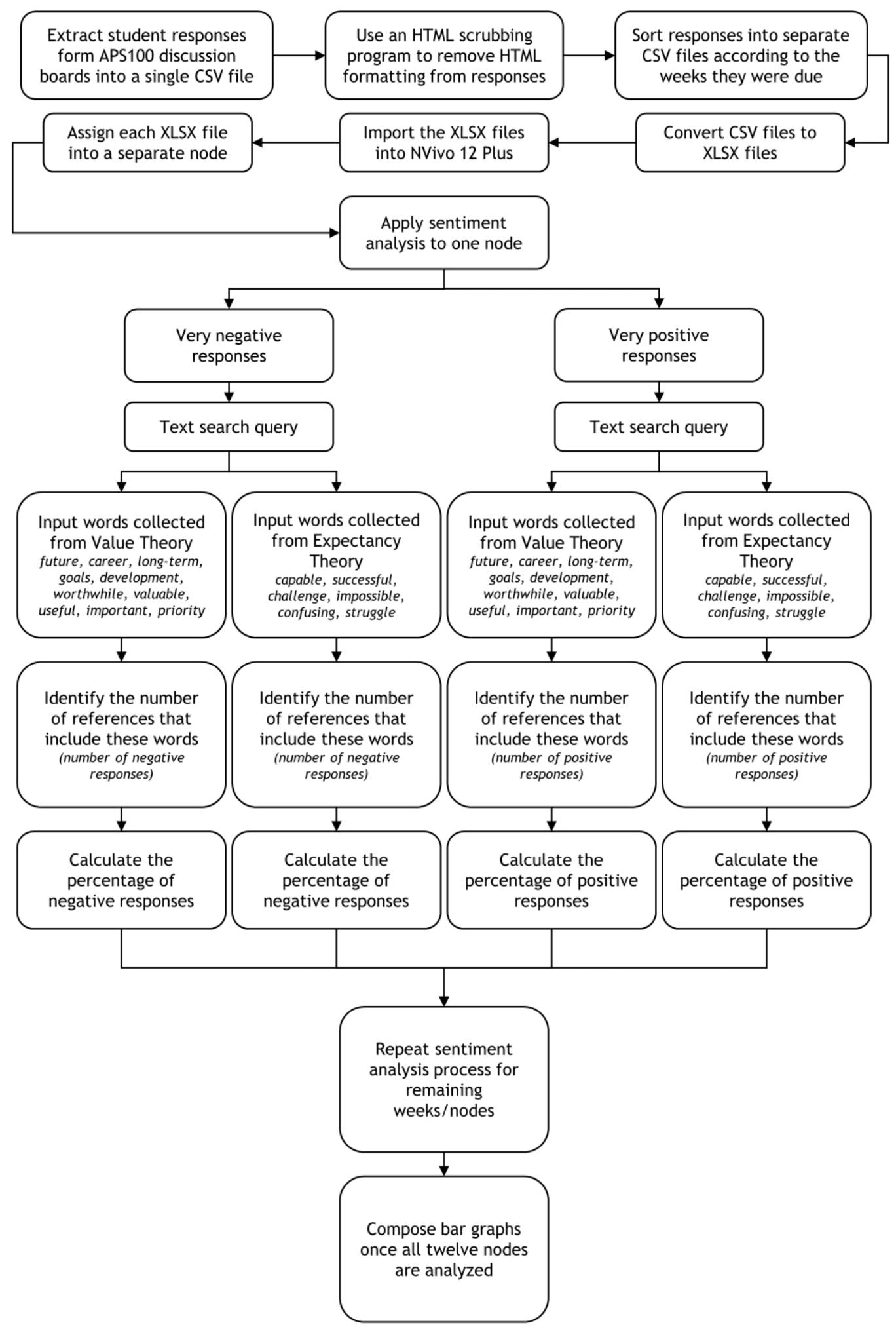

Fig. 2. This flow chart is a visualization of the data processing methodology from data extraction to result compilation. 
The data collected is sourced from the weekly discussion boards with approval from the University of Toronto's Research Ethics Board. Data collection begins after the course concludes. The information is collected from the discussion boards present on the Canvas LMS page and is stored in a CSV file. The original code that does this [12] is altered so that information linked to student identities is not imported. This ensures that responses are kept anonymous and cannot be traced to their authors [refer to Appendix A for the code alterations and Appendix B.1 for sample data]. A scrubbing program [refer to Appendix C] then removes HTML formatting from the data. All information enclosed by " $<$ " and "> symbols is removed [refer to Appendix B.2]. The scrubbed responses are then sorted into separate CSV files by the week they were assigned [refer to Appendix B.3]. The CSV data files are then converted to XLXS files and each file is imported as a separate "node" in NVivo.

The following process is applied to each node separately starting with the node containing responses from week one. Sentiment analysis is applied to the node using NVivo which outputs sorted collections of responses the software deems as having very positive, moderately positive, moderately negative, and very negative sentiment. A text search query is then applied to the very positive and very negative response collections. Collections of moderately negative and moderately positive responses are ignored as these responses contain sections with both positive and negative sentiment. Although this may also occur in responses deemed as very positive/negative, the conflict is considered to be inconsequential to the overall sentiment of the response.

Words drawn from the value component of EVT (refer to Fig. 1) are searched, and the number of references (the number of times the words appeared in the responses) are recorded. Another text search query is completed for the very positive and very negative responses, but instead, using the words drawn from the expectancy component of EVT (refer to Fig. 1). The references are recorded again. The files sorted into the sentiment nodes are then cleared, and this process is repeated for the remaining nodes (refer to Fig. 2 for a visualization). Once all the weeks are complete, the percentage of negative and positive references is calculated.

\section{RESULTS}

The graphs below (Fig.3 and Fig. 4) display the percentage of student responses classified as either very positive or very negative sentiment. Tables with the exact number of references and exact percentage values are provided in Appendix D.1 and Appendix D.2 respectively. The words drawn from the value component of the framework were found more in very positive responses compared to very negative. The lowest percentage of positive responses, and in consequence, highest percentage of negative responses, were drawn from the fourth week. In regard to the expectancy component of the framework, responses fluctuated between higher percentages of very positive and very negatives sentiment. The highest percentage of positive responses occurred during the seventh week, and the highest percentage of negative responses during the eighth. These conclusions can be used to identify possible correlations to intrinsic motivations of first-year engineering students.

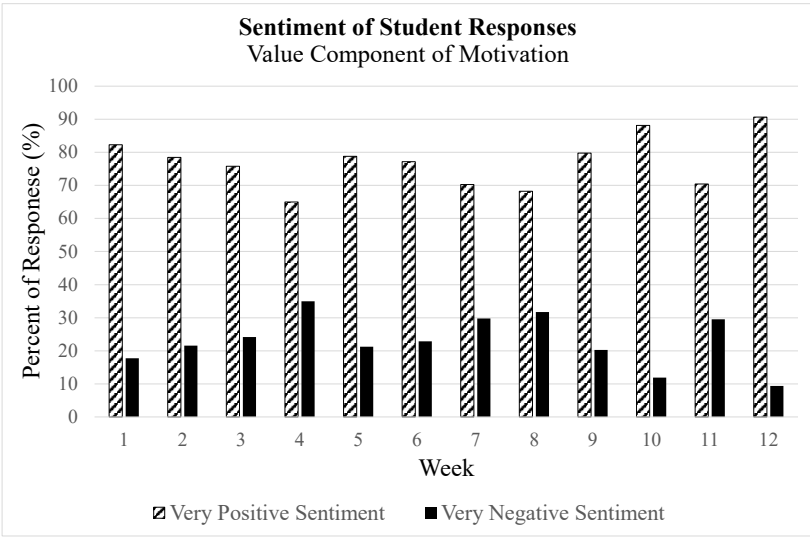

Fig 3. This graph displays the percentage of student responses deemed as very negative or very positive, using words drawn from the value component of the framework.

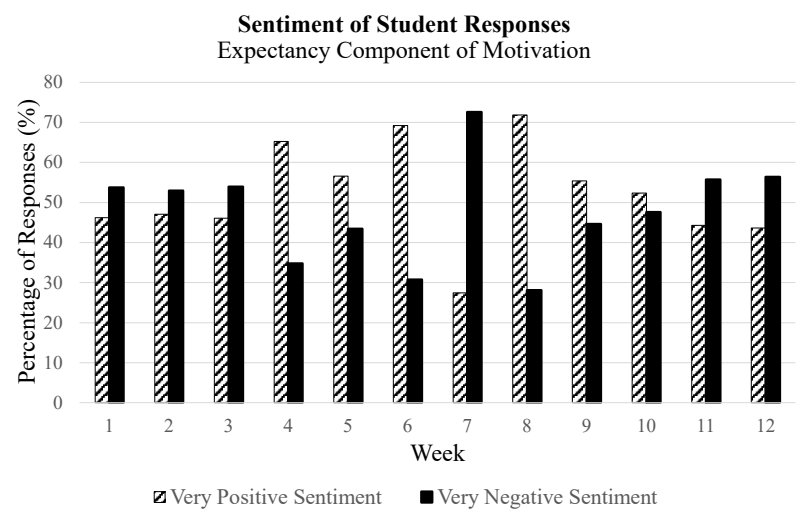

Fig 4. This graph displays the percentage of student responses deemed as very negative or very positive, using words drawn from the expectancy component of the framework. 


\begin{tabular}{|c|ccc|}
\cline { 2 - 5 } \multicolumn{1}{c|}{} & \multicolumn{4}{c|}{ Student Motivation Throughout the Fall 2020 Academic Term } \\
\hline Week & Value Component of Motivation & Expectancy Component of Motivation & Overall Motivation \\
1 & Mostly Positive & Mostly Negative & No Conclusion \\
2 & Mostly Positive & Mostly Negative & No Conclusion \\
3 & Mostly Positive & Mostly Negative & No Conclusion \\
4 & Mostly Positive & Mostly Positive & Relatively Positive \\
5 & Mostly Positive & Mostly Positive & Relatively Positive \\
6 & Mostly Positive & Mostly Positive & Relatively Positive \\
7 & Mostly Positive & Mostly Negative & No Conclusion \\
8 & Mostly Positive & Mostly Positive & Relatively Positive \\
9 & Mostly Positive & Mostly Positive & Relatively Positive \\
10 & Mostly Positive & Mostly Positive & Relatively Positive \\
11 & Mostly Positive & Mostly Negative & No Conclusion \\
12 & Mostly Positive & Mostly Negative & No Conclusion \\
\hline
\end{tabular}

Fig 5. This table displays the overall sentiment of student responses which include words related to EVT.

When identifying the trends in the data, weeks four to six and eight to ten have an overall positive sentiment. Relating this to the EVT, a relatively positive sentiment may correlate to higher levels of motivation during those weeks.

\section{DISCUSSION}

\subsection{Understanding the trends in motivation}

Referring to Fig.5, the motivation of students related to each component of the EVT is deemed as mostly positive or mostly negative depending on the percentage of responses with that assigned sentiment. If the week has a percentage of very positive responses greater than 50, that week is considered mostly positive. If the week has a percentage of very negative responses greater than 50, that week is considered mostly negative. For the overall motivation, the sentiment from both the value and expectancy components are analyzed. If both components are deemed mostly positive, the overall student motivation for that week is considered relatively positive. If the results differ in sentiment, no conclusions are drawn.

Results of the analyses describe the relative positive and negative sentiment regarding the components of motivation on a weekly basis. A mostly positive sentiment in the value component would indicate that the student body feels their productive situation (e.g., tasks, experiences, etc.) is valuable. A mostly positive sentiment for the expectancy component indicates that students feel that they are likely to succeed in their current situation. The inverse applies if the sentiment is mostly negative. Applying EVT to these results provides the insight that if the majority of the sentiment is positive in both the expectancy and value components for any given week, students are experiencing relatively high levels of motivation that week. Likewise, predominantly negative sentiments indicate low levels of motivation. Other combinations of sentiment do not clearly indicate the level of student motivation but show how students interpret the current situation.

Weeks four to six and eight to ten have an overall positive sentiment. This indicates that students may have higher levels of motivation to perform well in their engineering education during these weeks. Another interesting point to note is that the value component of EVT has a predominantly positive sentiment throughout the semester while the sentiment of the expectation component fluctuates. This indicates that students consistently find their engineering instruction and coursework to be valuable and worthwhile. However, the students do not always feel they are able to achieve success in the program.

Identifying these trends in motivation using EVT as the framework provides potential areas of improvement in the (online) engineering education context. Finding that the expectancy component of motivation procures a mostly negative sentiment throughout the term, suggests that implementing new or improved methods to support student success and strengthen student's belief that they are able to achieve success in their studies may be of interest. Furthermore, this paper provides a foundation for further work to be done in this field and provides sources of error to consider in future work.

\subsection{Sources of Error}

During preprocessing, HTML formatting was removed from the text in order to eliminate words that were not written by students. The method by which this was conducted however allowed for the possibility of valuable data loss if any student incorporated the " $<$ " symbol in their response. Intra-response data loss would occur when a student typed in a "<" symbol but a "> " symbol was present later in the response. Inter-response data loss would occur if a student typed a " $<$ " symbol in their response and the next ">" symbol was found in another student's response. When examining the scrubbed data, the number of responses recorded was the same as before scrubbing confirming that inter-response data loss did not occur. It was not feasible to ascertain whether intra-response data loss was present, so it remains a possible source of error.

Using the current method of chronologically sorting the data according to due date introduces error as some students may have submitted responses after the week that the discussions were due. The course instructor did give the assurance that an overwhelming majority of responses were submitted during the prescribed week, but this evidence is anecdotal. This error could have been avoided if the data were originally sorted based on the time of submission rather than the prescribed due date.

Another source of potential error arises from a few individual prompts encouraging biased responses as described in Section 1.4. This, however, does not occur every week and in any given week, the biased prompts only make up a quarter of all prompts available. It can be argued that the biased prompts may be more popular and therefore have greater influence over the result. The extent to which this is true could be statistically analyzed although this analysis was not conducted for this report. The presence of 
these few biased prompts was accepted in favour of the diversity the responses reflected and the sheer volume at which they were present.

Errors are also present in the sentiment analysis of the data. When using sentiment analysis in NVivo, sentences are searched for particular words that are deemed either to have inherent positive or negative sentiment [13]. If the words drawn from the expectancy and value theory, already have a positive or negative sentiment attached to them (i.e., they are not neutral in sentiment) possible inaccuracies may arise. The influence of this error cannot be gauged as QSR International (the company behind NVivo's development) does not release a word bank of which words the software considers as bearing positive/negative sentiment. Additionally, this version of NVivo cannot recognize, and therefore properly interpret, slang, double negatives, sarcasm, dialect variations, idioms or ambiguity present in the text [13]. The significance of this error can be reduced by using multiple sentiment analysis software provided by different companies and then averaging the results.

\section{CONCLUSIONS \& FUTURE WORK}

The sentiment of student responses, which included words drawn from the EVT, is found in this work. Using EVT as the framework for this research, higher levels of motivation are related to higher percentages of positive sentiment from both the value and expectancy components. However, with no conclusions being found for six out of the twelve weeks, there remains much improvement in this area of study. Future work may include identifying methods to mitigate the potential sources of error included in this process. Additionally, findings using EVT can be compared to findings using the Self-Determination Theory. Additionally, trends in motivation found from this research can be compared to student workload calendars. By doing so, possible correlations between presumed workload and motivational levels may be found.

\section{References}

[1] C. Variawa, APS100H1 F: Orientation to Engineering Course Syllabus for Fall 2020, University of Toronto, 2020.

[2] M. Cowley, "Students report less motivation, higher stress levels with the transition to online learning," North Texas Daily, 2019. [Online]. Available: https://www.ntdaily.com/students-report-lessmotivation-higher-stress-levels-with-the-transitionto-online-learning/. [Accessed January 2021].

[3] SAS Institute, "What is Natural Language Processing?," SAS Institute Inc., 2021. [Online]. Available:

https://www.sas.com/en_ca/insights/analytics/whatis-natural-language-processing- nlp.html\#: :text=Natural\%20language \%20processi ng\%20helps \%20computers, determine \%20which $\% 2$ 0parts\%20are\%20important. [Accessed January 2021].

[4] T. Mills, "What Is Natural Language Processing And What Is It Used For?," Forbes, 2 July 2018. [Online]. Available:

https://www.forbes.com/sites/forbestechcouncil/201 8/07/02/what-is-natural-language-processing-andwhat-is-it-used-for/?sh=1176a0165d71. [Accessed January 2021].

[5] M. D. Svinicki, A Guidebook On Conceptual Frameworks For Research In Engineering Education, University of Texas, 2010.

[6] T. A. R. F. S. G. C. K. M. J. C. M. Jacquelynne S. Eccles, "Expectancies, values, and academic behavior," Achievement and Achievement Motivation, 1983.

[7] R. V. L. P. R. R. E. L. Deci, "Motivation and education: The self-determination perspective," Educational Psychologist, vol. 26, no. 3-4, pp. 325346, 1991.

[8] K. Cherry, "What Is Motivation?," Verywell Mind, 27 April 2020. [Online]. Available: https://www.verywellmind.com/what-ismotivation-2795378. [Accessed January 2021].

[9] J. Clear, "Motivation: The Scientific Guide on How to Get and Stay Motivated," 11 November 2020. [Online]. Available: https://jamesclear.com/motivation. [Accessed January 2021].

[10] Sift, "What are Motivational Theories?," Sift, 2021. [Online]. Available: https://www.hrzone.com/hrglossary/what-are-motivational-theories. [Accessed January 2021].

[11] J. R. G. Allan Wigfield, "What Does Expectancyvalue Theory Have to Say about Motivation and Achievement in Times of Change and Uncertainty?," Motivation in Education at a Time of Global Change, vol. 20, pp. 15-32, 2019.

[12] A. M. Justin Lee, " ubccapico/canvas-discussion," GitHub, 6 November 2020. [Online]. Available: https://github.com/ubccapico/canvas-discussion.git. [Accessed 6 November 2020].

[13] QSR International, "Automatically detect and code sentiment," QSR International, 2018. [Online]. Available: https://helpnv.qsrinternational.com/12/win/v12.1.98d3ea61/Content/coding/auto-detect-codesentiment.htm. [Accessed January 2021]. 


\section{APPENDIX A: CHANGES MADE TO HTML EXTRACTION CODE}

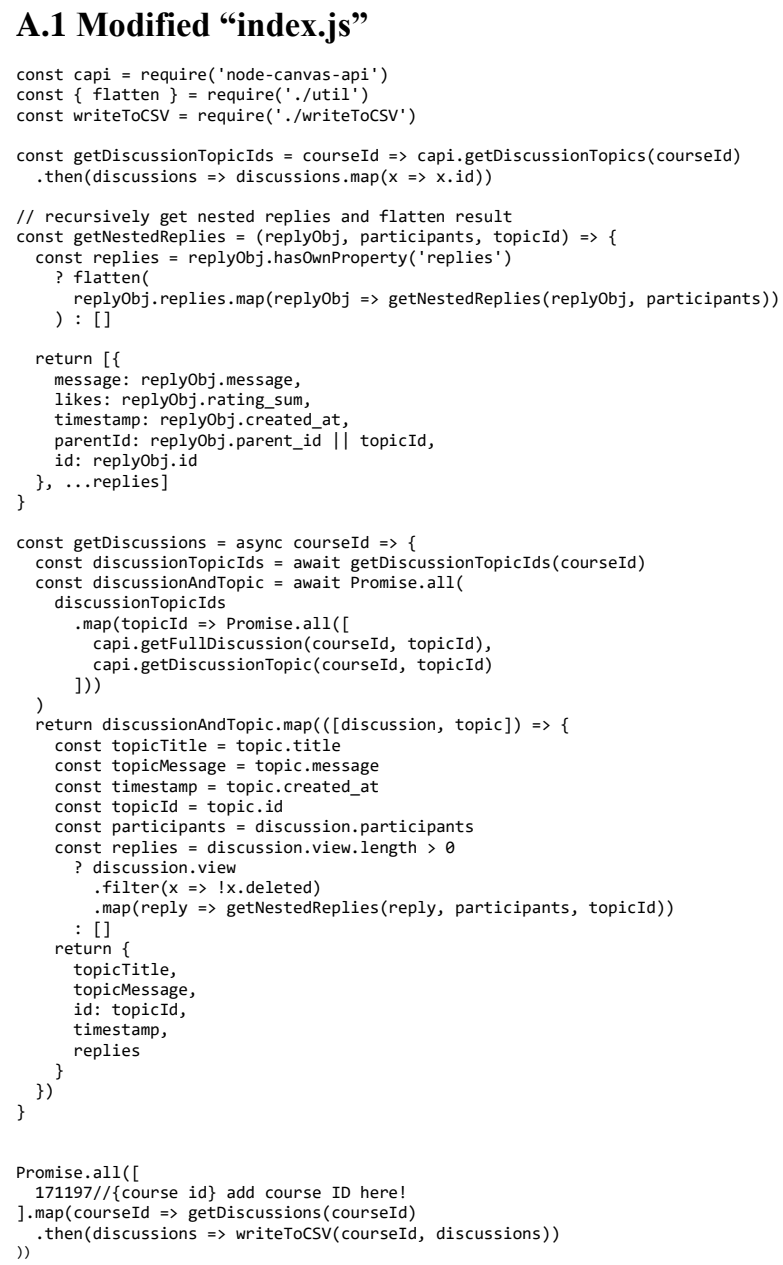

\section{A.2 Modified "util.js"}

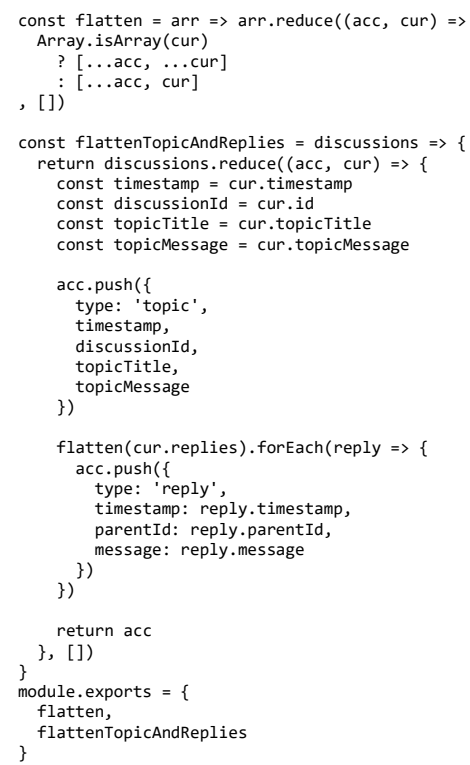

\section{A.2 Modified "writeToCSV.js"}

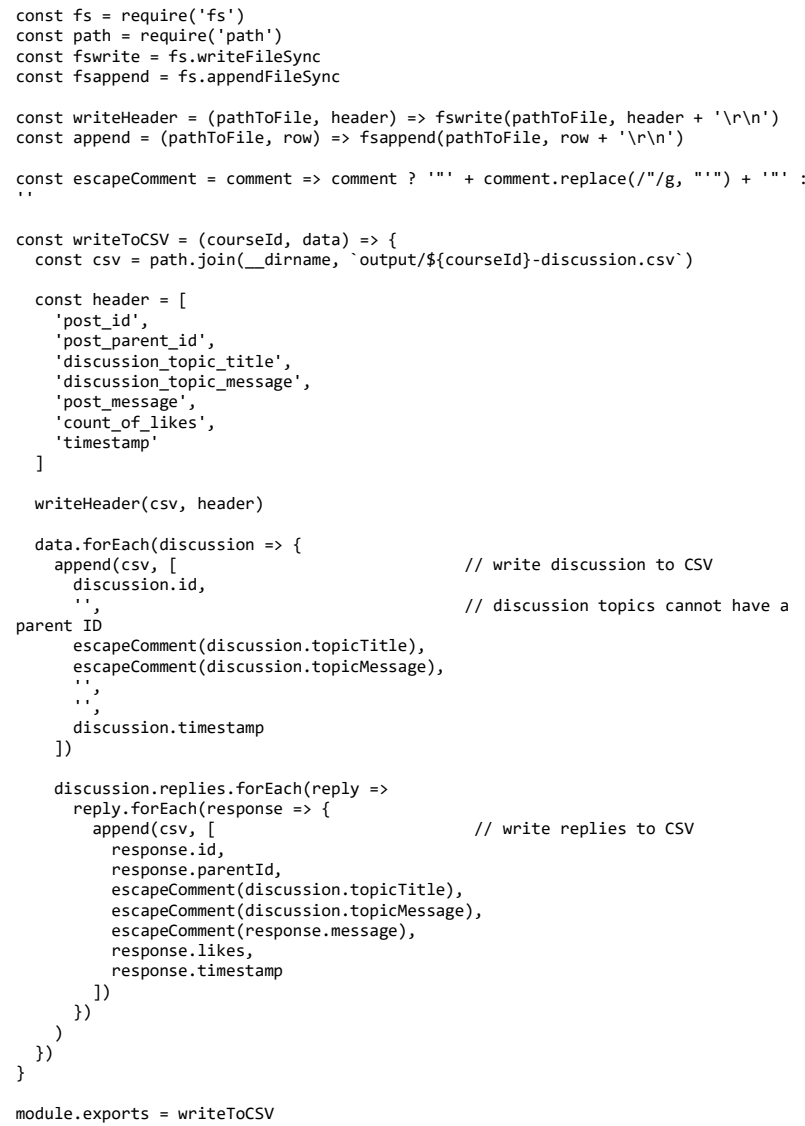

\section{APPENDIX B: DATA}

\section{B.1 Raw Data}

A sample of data as recoded by the data collected program.

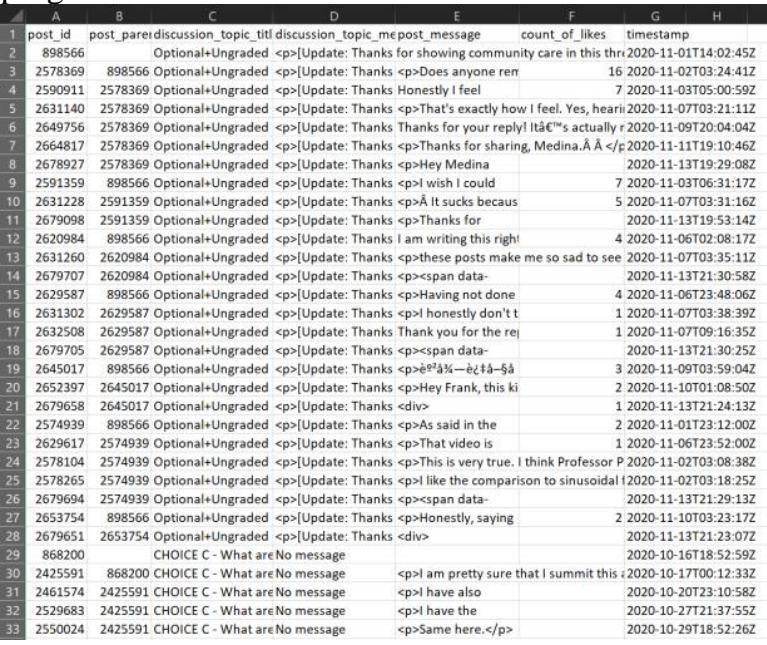




\section{B.1 Scrubbed Data}

A sample of data after being processed by the HTML scrubbing program.

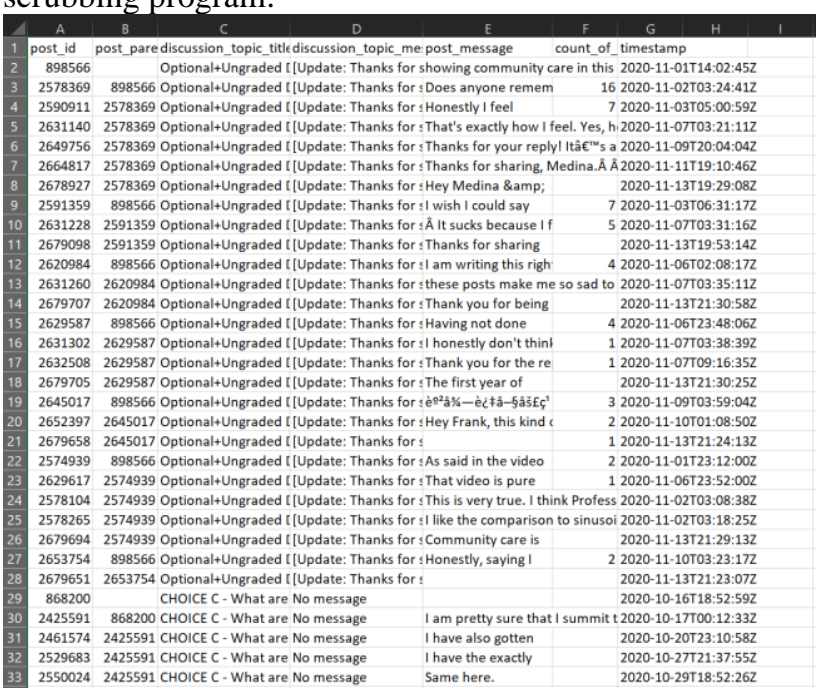

\section{APPENDIX C: CODE FOR HTML SCRUBBING PROGRAM}

\#include 〈stdio.h>

\#include <stdlib.h>

\#include 〈string.h>

\section{\#define MAXLENGTH 65535}

\#define MEMORY 1000001

void AppendFile (FILE* FilePtr, char ListofChar[1000001], int StopPosition); char ReadCharFromFile (FILE* FilePtr, int OffsetFromStart);
bool DetectFileEnd (FILE* FilePtr, long int offsetFromstart)

int main(void) \{

FILE* Tempile;

FILE* TargetFile;

char* TargetFileAddress $=($ char $*$ ) calloc $($ MAXLENGTH+1, $\operatorname{sizeof}($ char $))$;

char* TempFileAddress = "TempTxtFile.txt";

char CharacterResevoir [MEMORY]

char CurrentChar $=$ ' $a$ ';

int Counter $=\theta$;

int Counter $=0$;
int LengthofAddress $=0$;
long int Cursor $=-1$;

long int Cursor $=-1$;
bool FileEnded $=$ false;

//Setting up the temporary fil

TempFile $=$ fopen $($ TempFileAddress, $" w+") ; / /$ Creates a blank text file where the filtered conetent is temporarily stored in the same directory as the program end

//If the file cannot be created, an error message will pop up and the program will if $($ TempFile $==$ NULL $)\{$ printf("Error -- Temporary file cannot be created! \n"); exit(1);

fclose(TempFile); //Close file for now

//Locating target file

printf("Please enter (without surrounding in quotations) the PATH (file address including file name) of the .txt file you want to clean (n");

fgets(TargetFileAddress, MAXLENGTH, stdin); //Gets PATH to .txt file from user

//Removing newline character from PATH

Counter $=\theta$;

while(TargetFileAddress[Counter] != ' $\left.\backslash \mathrm{n}^{\prime}\right)$ \{

\}

TargetFileAddress [Counter] $=\cdot\left(\bullet \theta^{\prime}\right.$;

//Array is shortened to conserve memory

Counter $=0$; the string

$$
\text { Counter++; }
$$

LengthofAddress $=$ Counter +1 TargetFileAddress
LengthofAddress*sizeof(char)); //Adjusts size of pointer to length of address (char*)realloc(TargetFileAddress, Targetfile $=$ fopen(TargetfileAddress, $" r ")$; //Opens uner input .txt file for

//If the file cannot be opened, an error message will pop up and the program will

if (TargetFile $==$ NULL $)\{$
printf("Error -- Fil

else\{

printf("Error -- File cannot be opened/found! \n");

printf("File found and opened succesfully! $\backslash n ")$;

fclose(TargetFile);

//Add an ending sequence to the file that is not expected to be written in the

TargetFile = fopen (TargetFileAddress, "a");

CharacterResevoir $[0]=$ ' I';

CharacterResevoir [1] $=$ ' 0 '

CharacterResevoir [2] = ',

CharacterResevoir [3] = 'o';

AppendFile(TargetFile, CharacterResevoir, 4);

fclose(TargetFile);

//The entire target file is copied to the temporary file character by character.

Any HTML formatting enclosed and including ' $\langle$ ' and ' $>$ ' is ommitted

printf("Starting HTML cleaning.... \n");

FileEnded = false;

Cursor $=-1$;

while (FileEnded $==$ false) \{

ResevoirCounter $=-1$;
TargetFile $=$ fopen (TargetFileAddress, $" r ")$;

while ((ResevoirCounter < (MEMORY-1)) \&\& (FileEnded $==$ false) $)\{/ /$ Keeps on reading characters from source and storing them in the array. Ends when array is full or end of file is reached.

Cursor++;

if (CurrentChar $\left.=={ }^{\prime}<'\right)\{/ /$ HTML formatting detected

while (CurrentChar $!='>$ ') \{//Skipping HTML formatting

\}

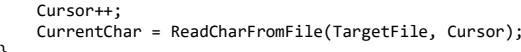$$
\begin{aligned}
& \text { Cursor++; } \\
& \text { CurrentChar }=\text { ReadCharFromFile }(\text { TargetFile, Cursor })
\end{aligned}
$$

else if ((CurrentChar $\left.==' \mid{ }^{\prime}\right)$ \& (DetectFileEnd(TargetFile, Cursor) $==$ true)) $\{/ /$ If first character of ending sequence is detected

to temp file Filended = true; //Stop the read cycle of target file and write cycle \}

else if ((CurrentChar $==$ ' $\backslash n ')$ \&\& (ReadCharFromfile(TargetFile, Cursor +1$)$ $\left.={ }^{\prime}\left(n^{\prime}\right)\right)\{/ /$ Accounts for the $C$ program reading the enter in the text file as two newline characters

ResevoirCounter++;

CharacterResevoir [ResevoirCounter] = 'In',

\} Cursor++; //Skips reading the next newline character

else $\{$ //Normal text is detected

CharacterResevoir[ResevoirCounter] = CurrentChar;$$
\text { \} }
$$

fclose(TargetFile); //Reading Sequence is ended

TempFile = fopen (TempFileAddress, "a");

AppendFile(TempFile, CharacterResevoir, ResevoirCounter); //Storing current resevoir into temp file

ose(TempFile);

printf("\%ld characters have been processed from the original file \n", Cursor);

TempFile $=$ fopen $($ TempFileAddress, $" a ") ; / /$ Adding ending sequence to temp file CharacterResevoir $[0]=$ ' $I$ ';

CharacterResevoir [1] = ' 0 ';

CharacterResevoir [2] = ',';

CharacterResevoir [3] = ' 0 ';
CharacterResevoir [4] = ' $\mid$ ';

AppendFile(TargetF
fclose (TempFile);

$\begin{aligned} & \text { printf( }(\text { "cle } \\ &\text { file... } \backslash n ") \text {. }\end{aligned}$

//Target file is wiped clean

TargetFile = fopen(TargetFileAddress, "w");

fclose(TargetFile);

//The temporary file will be copied back over to the target file but the ending sequence will be ommitted
FileEnded = false;$$
\text { Cursor = -1; }
$$

while (FileEnded $==$ false)

ResevoirCounter $=-1$

TempFile $=$ fopen (TempFileAddress, $" r ")$;

while ((ResevoirCounter < (MEMORY-1)) \&\& (FileEnded $==$ false $))\{/ /$ Keeps on reading characters from source and storing them in the array. Ends when array is full or end of file is reached.

CurrentChar = ReadCharFromfile(TempFile, Cursor);

if $(($ Currentchar $==\mid l ')$ \&\& (DetectFileEnd(TempFile, Cursor $)==$ true $))\{$ //If first character of ending sequence is detected

to target file 
else if ((CurrentChar $==$ ' $\left.^{\prime} n^{\prime}\right)$ \&\& (ReadCharFromFile(TempFile, Cursor +1$)=$ $\left.\left(n^{\prime}\right)\right)$ \{//Accounts for the C program reading the enter in the text file as two newlin characters

ResevoirCounter++; Cursor++; //Skips reading the next newline character

else $\{/ /$ Normal text is detected

CharacterResevoir [ResevoirCounter $]=$ CurrentChar \}

fclose(TempFile); //Reading Sequence is ended

TargetFile $=$ fopen $($ TargetFileAddress, $" \mathrm{a} ")$,

AppendFile(TargetFile, CharacterResevoir, ResevoirCounter); //Storing current data in resevoir into temp file

printf("\%ld characters have been transferred to the original file $\backslash n "$, Cursor)

printf("HTML cleaning complete! \n");

//Remove temporary file and end program

if $($ remove (TempFileAddress) $==\theta)\{$
printf("Temproray file deleted successfully $\backslash n ")$,

else

printf("Temproray file deleted successfully\n");

printf("Unable to delete temporary file. F

\}

printf("Ending program....n");

\}

void AppendFile (FILE* FilePtr, char ListofChar[MEMORY], int StopIndex) \{

for (int $i=0 ; i<=S t o p I n d e x ; i++)\{$

\}

fprintf(FilePtr, "\%", ListofChar[i]);

return;

char ReadCharFromfile (FILE* FilePtr, int offsetFromstart) \{

char Output $=$ ' $a$ '

fseek(FilePtr, offsetFromStart, SEEK_SET);//Brings cursor to current position of interest

fscanf(FilePtr, "\%c", \&output).

bool DetectFileEnd (FILE* FilePtr, long int OffsetFromstart) \{

bool Output = false;

char CurrentChar $=$ ' $a$ ';

long int Offset $=$ OffsetFromstart;

CurrentChar $=$ ReadCharFromfile(FilePtr, offset $)$;

//Testing for the ending sequence

if (CurrentChar $==$ ' $\mid$ ')

CurrentChar $=$ ReadCharFromFile(FilePtr, Offset $)$

if (CurrentChar $==$ ' 0 ')

Offset++,

CurrentChar $=$ ReadCharFromfile(FilePtr, offset $)$;

if (CurrentChar $==$ ' ' ')

Offset++;

CurrentChar $=$ ReadCharFromfile(FilePtr, offset $)$;

if (CurrentChar $\left.=={ }^{\prime} \mathrm{O}{ }^{\prime}\right)\{$

Offset++;

CurrentChar $=$ ReadCharFromfile(FilePtr, offset $)$;

if (Currentchar $==' \mid$ ') \{

else

\{

$$
\text { , }
$$$$
\text { Output }=\text { false }
$$

else \{

els

$$
\text { \} }
$$$$
\text { Output }=\text { false; }
$$

else \{

\}

else \{

else \{

Output $=$ false;

Output $=$ false;
APPENDIX D: RESULTS

\section{D.1 Number of References Based on Sentiment}

\begin{tabular}{|c|ccc|}
\cline { 2 - 4 } \multicolumn{1}{c|}{} & \multicolumn{3}{c|}{ Value Component of Motivation } \\
\hline Week & Very Positive References & Very Negative References & Total References \\
1 & 116 & 25 & 141 \\
2 & 171 & 47 & 218 \\
3 & 116 & 37 & 153 \\
4 & 78 & 42 & 120 \\
5 & 141 & 38 & 179 \\
6 & 27 & 8 & 35 \\
7 & 78 & 33 & 111 \\
8 & 114 & 53 & 167 \\
9 & 122 & 31 & 153 \\
10 & 156 & 21 & 177 \\
11 & 93 & 39 & 132 \\
12 & 291 & 30 & 321 \\
\hline
\end{tabular}

\begin{tabular}{|c|ccc|}
\cline { 2 - 4 } \multicolumn{1}{c|}{} & \multicolumn{3}{c|}{ Expectancy Component of Motivation } \\
\hline Week & Very Positive References & Very Negative References & Total References \\
1 & 43 & 50 & 93 \\
2 & 64 & 72 & 136 \\
3 & 35 & 41 & 76 \\
4 & 139 & 74 & 213 \\
5 & 43 & 33 & 76 \\
6 & 9 & 4 & 13 \\
7 & 14 & 37 & 51 \\
8 & 23 & 9 & 32 \\
9 & 67 & 54 & 121 \\
10 & 22 & 20 & 42 \\
11 & 31 & 39 & 70 \\
12 & 24 & 31 & 55 \\
\hline
\end{tabular}

\section{D.2 Percentage of References Based on Sentiment}

\begin{tabular}{|c|cc|}
\cline { 2 - 3 } \multicolumn{1}{c|}{} & \multicolumn{2}{c|}{ Value Component of Motivation } \\
\hline Week & Very Positive References (\%) & Very Negative References (\%) \\
1 & 82.27 & 17.73 \\
2 & 78.44 & 21.56 \\
3 & 75.82 & 24.18 \\
4 & 65 & 35 \\
5 & 78.77 & 21.23 \\
6 & 77.143 & 22.86 \\
7 & 70.27 & 29.73 \\
8 & 68.26 & 31.74 \\
9 & 79.74 & 20.26 \\
10 & 88.14 & 11.86 \\
11 & 70.46 & 29.54 \\
12 & 90.65 & 9.35 \\
\hline
\end{tabular}

\section{Value Component of Motivation}

\begin{tabular}{|c|cc|}
\hline Week & Very Positive References (\%) & Very Negative References (\%) \\
1 & 46.24 & 53.76 \\
2 & 47.06 & 52.94 \\
3 & 46.05 & 53.95 \\
4 & 65.26 & 34.74 \\
5 & 56.58 & 43.42 \\
6 & 69.23 & 30.77 \\
7 & 27.45 & 72.55 \\
8 & 71.88 & 28.12 \\
9 & 55.37 & 44.63 \\
10 & 52.38 & 47.62 \\
11 & 44.29 & 55.71 \\
12 & 43.64 & 56.36 \\
\hline
\end{tabular}

